\title{
Compact Wideband Bandpass Filters Using Stepped-Impedance Resonators and Interdigital Coupling Structures
}

\author{
Cheng-Hsien Liang and Chi-Yang Chang, Member, IEEE
}

\begin{abstract}
This letter proposes a microstrip $\lambda / 4$ interdigital stepped-impedance resonator whose impedance ratio can be very low and coupling strength between adjacent resonators can be very large. On the basis of the proposed structure, one four-pole Chebyshev and one four-pole generalized Chebyshev filter with $0.05 \mathrm{~dB}$ equal-ripple bandwidths of $48 \%$ and $46 \%$, respectively, are designed and fabricated. Both implemented filters have a compact size, a wide passband, a wide upper stopband, and a high spurious passband at near 5.1 times the center frequency. Good agreement between measurements and simulations is observed.
\end{abstract}

Index Terms-Interdigital coupled lines, microstrip, steppedimpedance resonator (SIR), wideband bandpass filter (BPF).

\section{INTRODUCTION}

$\mathbf{R}$ ECENTLY, planar filters with the characteristics of low cost, compact size, and wide stopband play an important role in modern filter applications due to easy integration into the printed circuit board (PCB). Moreover, next generation wireless systems and high data-rate communication systems require wideband bandpass filters (BPFs). For wideband applications, the conventional edge-coupled BPFs [1] require strong coupling between adjacent resonators, which requires close spacing and leads to fabrication difficulty. Broadside coupled structures [2]-[5] enable stronger coupling and filters with these structures exhibit inherently wideband characteristics. Other techniques such as three-line microstrips [6], multimode resonators [7], [8], the cascade of lowpass and highpass filters [9], and the new coupling scheme in [10] are used to design wideband BPFs. However, the above-mentioned filters may still be large in size or have a narrow upper stopband.

The stepped-impedance resonator (SIR) has advantages to reduce the circuit size and to improve the upper stopband performance [11]. Theoretical analysis reveals that the impedance ratio $R=Z_{L} / Z_{H}$ is the most important parameter characterizing the properties of the SIR, where $Z_{H}$ and $Z_{L}$ are the characteristic impedances of the high- and low-impedance sections,

Manuscript received March 16, 2009; revised May 14, 2009. First published August 11, 2009; current version published September 02, 2009. This work was supported in part by the National Science Council under Grant NSC95-2221-E009-042-MY3, and by the Ministry of Education (MoE) under an MoE Aiming for the Top University (ATU) Plan Grant.

The authors are with the Department of Communication Engineering, National Chiao Tung University, Hsinchu 300, Taiwan (e-mail: seaman. cm96g@g2.nctu.edu.tw; mhchang@cc.nctu.edu.tw).

Color versions of one or more of the figures in this paper are available online at http://ieeexplore.ieee.org.

Digital Object Identifier 10.1109/LMWC.2009.2027060

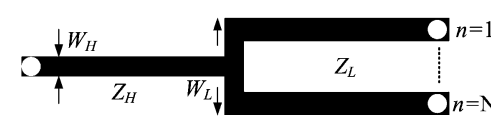

(a)

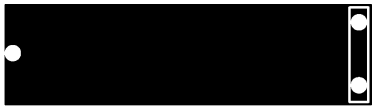

(b)
Fig. 1. Proposed SIR configuration. (a) Top view. (b) Bottom view $(N=2)$.

respectively. Since the $\lambda / 4$ SIR has a smaller size and fewer spurious modes than the $\lambda / 2$ SIR, it is suitable for microstrip filters. For the SIR structure, the lower the impedance ratio $R$ is, the shorter the resonator length and the farther the first spurious resonant frequency will be. As a result, for the conventional SIR with a low impedance ratio $R$, it is difficult to obtain enough coupling for wideband BPFs.

In this letter, two compact wideband BPFs based on the proposed $\lambda / 4$ interdigital SIR are presented. By using interdigital coupled lines, strong coupling between adjacent resonators is achieved. In the meantime, the impedance ratio $R$ of the proposed $\lambda / 4$ interdigital SIR is still kept low so as to reduce the filter size and to extend the upper stopband. Furthermore, by introducing cross coupling between nonadjacent resonators, a pair of transmission zeros on both sides of the passband can be easily obtained to improve the selectivity of the proposed four-pole wideband BPFs.

\section{Four-Pole Chebyshev Filter}

Fig. 1 shows the top and bottom layouts of the proposed $\lambda / 4$ interdigital SIR. Specifically, the proposed $\lambda / 4$ SIR consists of several parallel-connected thin strips to form the low-impedance section $\left(Z_{L}\right)$ and a short-circuited thin strip to form the highimpedance section $\left(Z_{H}\right)$. Note that in Fig. 1(a), via-holes are applied on the open end of each strip in the low-impedance section where these via-holes are connected through a thin strip on the bottom layer, as shown in Fig. 1(b). This structure enables the resonator to maintain the low impedance ratio $R$ and to have better spurious response. Due to the slots in the low-impedance section of the proposed SIR, the impedance ratio $R$ is slightly larger than that of the conventional microstrip SIR without slots.

The proposed four-pole filter I is a Chebyshev filter with a passband ripple of $0.05 \mathrm{~dB}$, a center frequency $\left(f_{0}\right)$ of $1.0102 \mathrm{GHz}$, and a fractional bandwidth of $48 \%$. The first spurious frequency is set more than $5 f_{0}$. Filter I was fabricated on a Rogers RO4003 substrate with a relative dielectric constant of 3.58, a loss tangent of 0.0027 , and a thickness of $0.508 \mathrm{~mm}$. According to the specifications, the filter is implemented using the design procedures based on the coupling coefficient $k$ and 


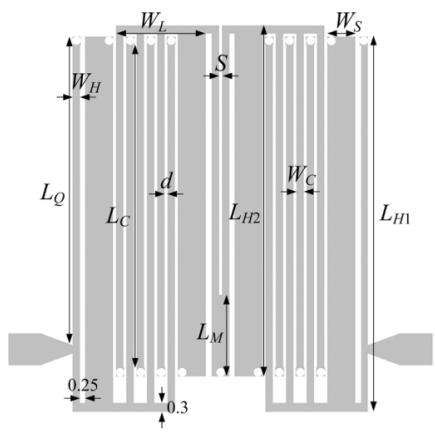

(a)

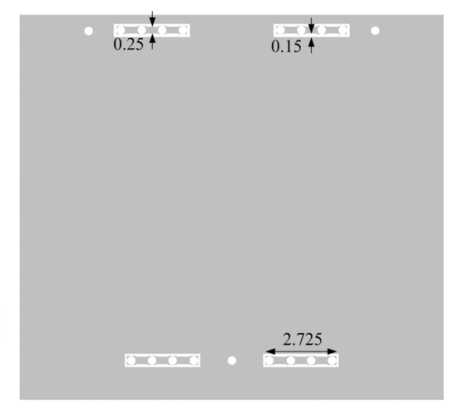

(b)
Fig. 2. Configuration of the proposed filter I. (a) Top-layer layout. (b) Bottomlayer layout. Filter dimensions: $L_{Q}=11.9, L_{H 1}=14.45, L_{H 2}=13.525$, $L_{C}=12.5, L_{M}=3.25, W_{H}=W_{C}=0.25, W_{L}=3.45, W_{S}=1.05$, and $S=d=0.15 \mathrm{~mm}$.

the external quality factor $Q_{e}[1]$. Here, $Q_{e}=1.9975, k_{12}=$ $k_{34}=0.4304$, and $k_{23}=0.3324$. The full-wave EM software Sonnet is employed in the simulation. The closed box in Sonnet is set large enough so that it has little effect on the measured data in the open environment.

Fig. 2 depicts the top and bottom layouts of filter I. Here, we number these four resonators as 1 to 4 from left to right. Each SIR is folded in order to apply the electric or magnetic coupling and to reduce the filter size. As shown in Fig. 2(a), the parallel-connected thin strips in the low-impedance section of the SIR are interdigitally coupled to the adjacent SIR. This forms a strong capacitive coupling between resonators 1 and 2 as well as 3 and 4 . The coupling strength is determined by the gap width $d$, the strip width $W_{C}$, the number $N$ of parallel-connected strips, and the interleave length $L_{C}$. By adjusting these parameters, various degrees of coupling between adjacent SIRs can be obtained. The strong coupling between resonators 2 and 3 is realized by the common transmission line connected to ground. The strength of the coupling is adjusted by the length $L_{M}$ of the common short-circuited stub. In this filter configuration, the coupling $k_{23}$ is magnetic, while $k_{12}$ and $k_{34}$ are mainly electric. The tap position $L_{Q}$ of the input and output feed lines is chosen to match the $Q_{e}$ value for the 50- $\Omega$ source/load impedance.

In the design process, we first fix the widths of $W_{H}, W_{C}, S$, and $d$, and then determine the number $N$ roughly for a given filter specification. $W_{S}$ (i.e., $n=1$ ) can be adjusted for the prescribed normalized spurious resonant frequency. Here, the resonator dimensions are $W_{H}=W_{C}=0.25 \mathrm{~mm}, S=d=$ $0.15 \mathrm{~mm}, W_{S}=1.05 \mathrm{~mm}, W_{L}=3.45 \mathrm{~mm}$, and $N=4$. All diameters of via-holes have the same size of $0.3 \mathrm{~mm}$. On the basis of the structures in Fig. 2, the design curves for the external quality factor and the coupling coefficients with $L_{Q}$, $L_{C}$, and $L_{M}$ as parameters are shown in Fig. 3, respectively.

Fig. 4 shows the fabricated filter $I$ with a size of $11.45 \mathrm{~mm} \times 14.9 \mathrm{~mm}$, which is $0.0655 \lambda_{g} \times 0.0853 \lambda_{g}$, where $\lambda_{g}$ is the guided wavelength of $50-\Omega$ line on the substrate at the center frequency. Fig. 5 illustrates its simulated and measured responses. The measured results show that the filter has a center frequency of $1.0105 \mathrm{GHz}$. The measured 3-dB fractional bandwidth is $64.83 \%$ from 0.7149 to $1.37 \mathrm{GHz}$. Within the passband, the minimum insertion loss is $0.63 \mathrm{~dB}$, and the return loss is better than $18.9 \mathrm{~dB}$. The first spurious response is at

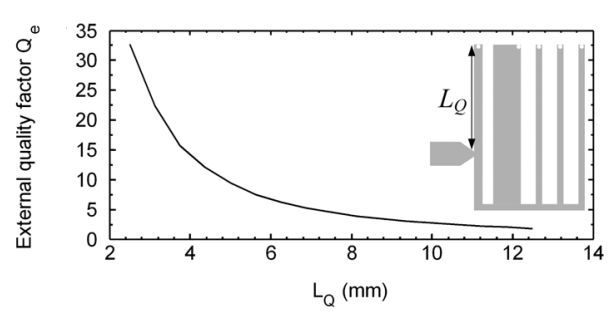

(a)

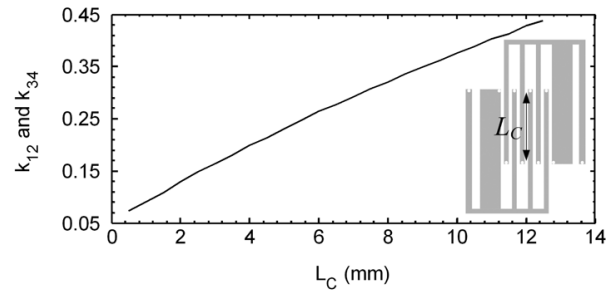

(b)

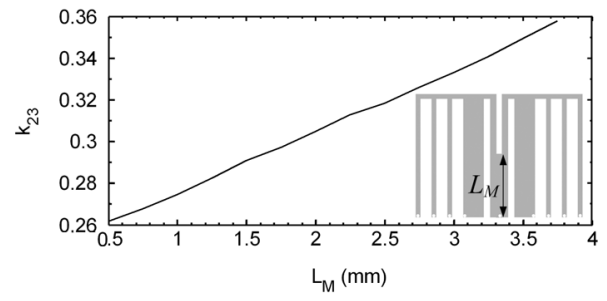

(c)

Fig. 3. Design curves for the proposed filter I. (a) External quality factor $Q_{e}$. (b) Electric coupling $k_{12}$ and $k_{34}$. (c) Magnetic coupling $k_{23}$.

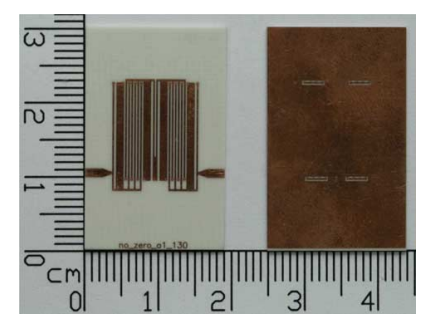

Fig. 4. Top view (left side) and bottom view of the fabricated filter I.

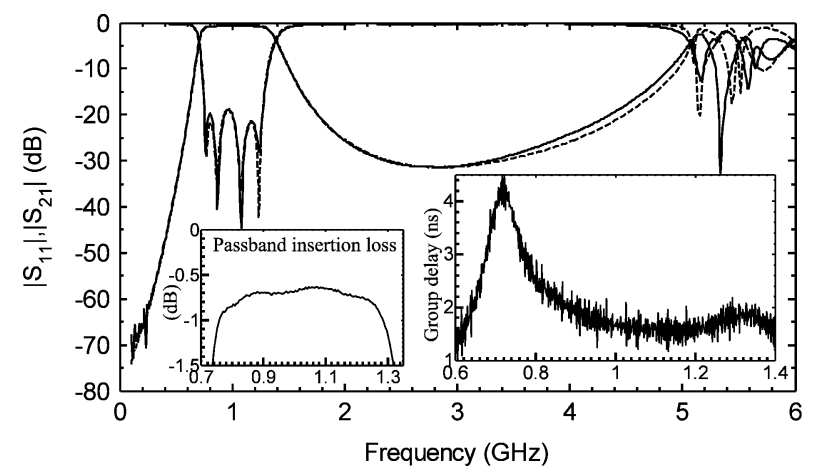

Fig. 5. Simulated (dashed line) and measured (solid line) results ( $\left|S_{11}\right|,\left|S_{21}\right|$, and group delay) of filter I. The $\mathrm{X}$-axis unit of both insets is $\mathrm{GHz}$.

$5.152 \mathrm{GHz}\left(=5.1 f_{0}\right)$, and the rejection level is better than $-20 \mathrm{~dB}$ from 1.76 to $4.357 \mathrm{GHz}$. 


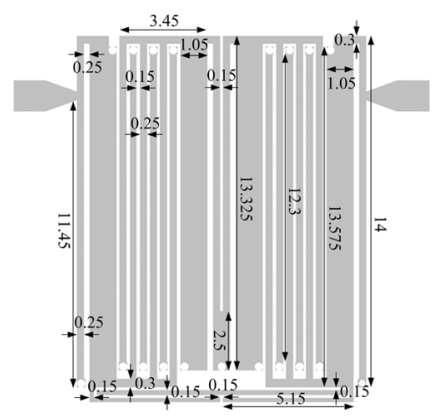

(a)

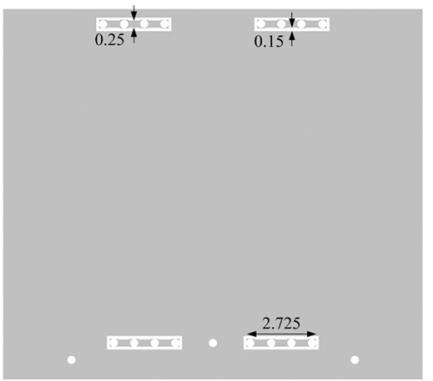

(b)
Fig. 6. Configuration of the proposed filter II. (a) Top-layer layout. (b) Bottomlayer layout. units: $\mathrm{mm}$.

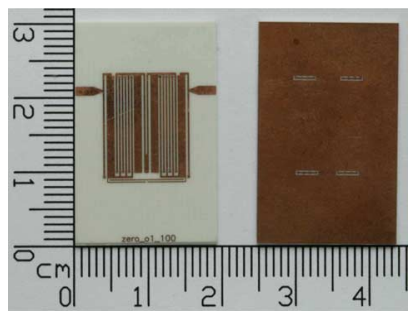

Fig. 7. Top view (left side) and bottom view of the fabricated filter II.

\section{Four-Pole GENERAlized CheByshev FILTER}

Since planar wideband Chebyshev BPFs usually have the poor upper stopband rejection, a generalized Chebyshev wideband BPF is proposed using the four-pole cross-coupled configuration. Fig. 6 depicts the top and bottom layouts of filter II. A thin microstrip line is applied at the bottom of the filter to provide the small cross coupling between resonators 1 and 4. This cross coupling produces a pair of transmission zeros on either side of the stopband. Because the cross coupling is weak, the initial design procedure is based on the fourth-order Chebyshev response filter with a $0.05 \mathrm{~dB}$ equal-ripple passband characteristic and follows the design process discussed above. After that, put a thin cross-coupling microstrip line and then slightly fine tune the whole filter. The proposed filter II is designed at a center frequency $\left(f_{0}\right)$ of $1.0079 \mathrm{GHz}$ with a fractional bandwidth of $46 \%$. Filter II was fabricated on the same substrate as filter I. Again, all the via-holes are $0.3 \mathrm{~mm}$ in diameter.

Fig. 7 shows the fabricated filter II with a size of $11.45 \mathrm{~mm} \times 14.6 \mathrm{~mm}$, i.e., $0.0654 \lambda_{g} \times 0.0833 \lambda_{g}$. The simulated and measured responses of filter II are presented in Fig. 8. The measured results show that the filter has a center frequency of $1.0076 \mathrm{GHz}$ and two transmission zeros at 0.3185 and $1.877 \mathrm{GHz}$. The measured 3-dB fractional bandwidth is $59.19 \%$ from 0.7256 to $1.322 \mathrm{GHz}$. The filter has a minimum insertion loss of $0.64 \mathrm{~dB}$ and a return loss better than $19.5 \mathrm{~dB}$ within the passband. The first spurious frequency is at $5.159 \mathrm{GHz}\left(=5.12 f_{0}\right)$. The rejection level is better than $-20 \mathrm{~dB}$ from 1.542 to $4.761 \mathrm{GHz}$. Apparently, the stopband depth is largely improved compared to that of filter I.

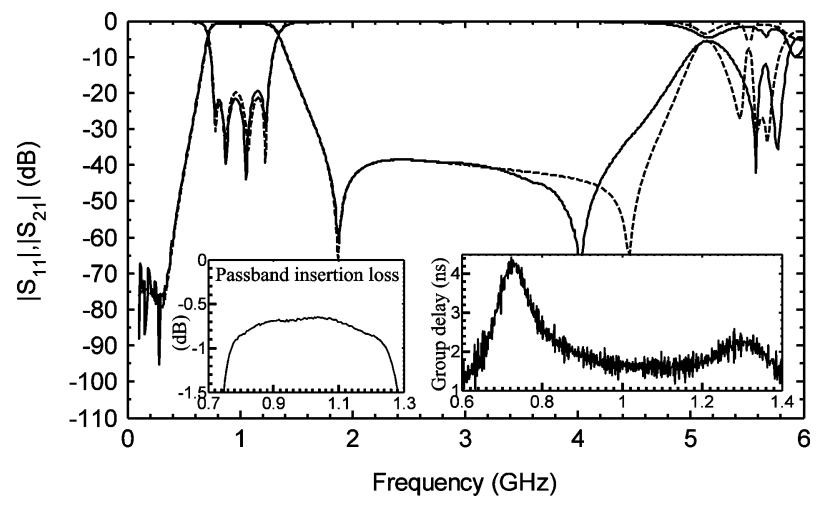

Fig. 8. Simulated (dashed line) and measured (solid line) results $\left(\left|S_{11}\right|,\left|S_{21}\right|\right.$, and group delay) of filter II. The $\mathrm{x}$-axis unit of both insets is GHz.

\section{CONCLUSION}

The proposed $\lambda / 4$ interdigital SIR has successfully solved the coupling strength problem of the conventional $\lambda / 4$ SIR. By using the proposed resonator, the BPF can have a wide passband and obtain a good upper stopband performance. Moreover, the filter fractional bandwidth is easily varied by changing the number of interdigital strips and the interleave length. The filter with a generalized Chebyshev response can be easily achieved by applying a thin cross-coupling strip. Both of the proposed filters have a very compact size, a wide passband, and a wide upper stopband.

\section{REFERENCES}

[1] J. S. Hong and M. J. Lancaster, Microstrip Filters for RF/Microwave Applications. New York: Wiley, 2001.

[2] M. Tran and C. Nguyen, "Modified broadside-coupled microstrip lines suitable for MIC and MMIC applications and a new class of broadsidecoupled band-pass filters," IEEE Trans. Microw. Theory Tech., vol. 41, no. 8, pp. 1336-1342, Aug. 1993.

[3] P. H. Deng, C. H. Wang, and C. H. Chen, "Novel broadside-coupled bandpass filters using both microstrip and coplanar-waveguide resonators," IEEE Trans. Microw. Theory Tech., vol. 54, no. 10, pp. 3746-3750, Oct. 2006.

[4] T. N. Kuo, S. C. Lin, C. H. Wang, and C. H. Chen, "Compact bandpass filters based on dual-plane microstrip/coplanar-waveguide structure with quarter-wavelength resonators," IEEE Microw. Wireless Compon. Lett., vol. 17, no. 3, pp. 178-180, Mar. 2007.

[5] M. K. Mandal and S. Sanyal, "Compact wide-band bandpass filter using microstrip to slotline broadside-coupling," IEEE Microw. Wireless Compon. Lett., vol. 17, no. 9, pp. 640-642, Sep. 2007.

[6] J. T. Kuo and E. Shih, "Wideband bandpass filter design with threeline microstrip structures," Proc. Inst. Elect. Eng., vol. 149, no. 56, pp. 243-247, Oct./Dec. 2002.

[7] W. Menzel, L. Zhu, K. Wu, and F. Bogelsack, "On the design of novel compact broad-band planar filters," IEEE Trans. Microw. Theory Tech., vol. 51, no. 2, pp. 364-370, Feb. 2003.

[8] Y. C. Chiou, J. T. Kuo, and E. Cheng, "Broadband quasi-Chebyshev bandpass filters with multimode stepped-impedance resonators (SIRs)," IEEE Trans. Microw. Theory Tech., vol. 54, no. 8, pp. 3352-3358, Aug. 2006.

[9] C. L. Hsu, F. C. Hsu, and J. T. Kuo, "Microstrip bandpass filters for ultra-wideband (UWB) wireless communications," in IEEE MTT-S Int. Dig., Jun. 2005, pp. 679-682.

[10] T. N. Kuo, S. C. Lin, C. H. Wang, and C. H. Chen, "New coupling scheme for microstrip bandpass filters with quarter-wavelength resonators," IEEE Trans. Microw. Theory Tech., vol. 56, no. 12, pp. 2930-2935, Dec. 2008.

[11] M. Makimoto and S. Yamashita, Microwave Resonators and Filters for Wireless Communication: Theory, Design and Application. Berlin, Germany: Springer-Verlag, 2001. 